\title{
Wavelength-resolved Purcell enhancement of PbS/CdS quantum dots measured on a chip-based platform
}

Elsinger, Lukas, Gourgues, Ronan, Zadeh, Iman, Maes, Jorick, Guardiani, Antonio, et al.

Lukas Elsinger, Ronan Gourgues, Iman E. Zadeh, Jorick Maes, Antonio Guardiani, Gabriele Bulgarini, Silvania F. Pereira, Sander N. Dorenbos, Val Zwiller, Zeger Hens, Dries Van Thourhout, "Wavelength-resolved Purcell enhancement of $\mathrm{PbS} / \mathrm{CdS}$ quantum dots measured on a chip-based platform," Proc. SPIE 11289, Photonic and Phononic Properties of Engineered Nanostructures X, 1128914 (26 February 2020); doi: 10.1117/12.2547265 


\title{
Wavelength-resolved Purcell enhancement of $\mathrm{PbS} / \mathrm{CdS}$ quantum dots measured on a chip-based platform
}

\author{
Lukas Elsinger $^{a},{ }^{b}$, Ronan Gourgues ${ }^{c}$, Iman E. Zadeh ${ }^{d}$, Jorick Maes ${ }^{e},{ }^{b}$, Antonio Guardiani $^{c}$, \\ Gabriele Bulgarini $^{c}$, Silvania F. Pereira ${ }^{d}$, Sander N. Dorenbos ${ }^{c}$, Val Zwiller ${ }^{f}$, Zeger Hens ${ }^{e}{ }^{b}$ and \\ Dries Van Thourhout ${ }^{a}{ }^{b}$ \\ ${ }^{a}$ Photonics Research Group, Ghent University - imec, 9052 Ghent, Belgium \\ ${ }^{b}$ NB Photonics, Ghent University, 9052 Ghent, Belgium \\ ${ }^{c}$ Single Quantum B.V., 2628CJ Delft, The Netherlands \\ ${ }^{d}$ Optics Research Group, Delft University of Technology, 2628 CJ Delft, The Netherlands \\ ${ }^{e}$ Physics and Chemistry of Nanostructures Group, Ghent University, 9000 Ghent, Belgium

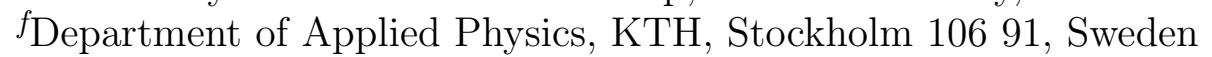

\begin{abstract}
Future quantum optical networks will require an integrated solution to multiplex suitable sources and detectors on a low-loss platform. Here we combined superconducting single-photon detectors with colloidal PbS/CdS quantum dots (QDs) and low-loss silicon nitride passive photonic components to show their combined operation at cryogenic temperatures. Using a planar concave grating spectrometer, we performed wavelength-resolved measurements of the photoluminescence decay of QDs, which were deterministically placed in the gap of plasmonic antennas, in order to improve their emission rate. We observed a Purcell enhancement matching the antenna simulations, with a concurrent increase of the count rate on the superconducting detectors.
\end{abstract}

Keywords: Colloidal Quantum Dots, Plasmonic Antennas, Superconducting Nanowire Single-Photon Detectors, Silicon Nitride, Photonic Integration

\section{INTRODUCTION}

Applications in quantum optics have stringent performance requirements on single-photon sources and detectors, in addition to the need for low-loss passive components. Integrating the building blocks on a chip-based platform can enable scaling up to larger experiments, whilst improving phase stability. A key property of single-photon sources is their radiative lifetime, which can be modified through the high local density of states (LDOS) created by plasmonic antennas, ${ }^{1}$ this is commonly referred to as "Purcell enhancement". In recently published work we demonstrated an up to 200-fold enhancement of the microsecond lifetime of IR-emitting colloidal PbS/CdS quantum dots (QDs), with a concurrent increase of the count rate. ${ }^{2}$

Here, we show first wavelength-resolved measurements of the Purcell enhancement of QDs deterministically positioned in the gap of plasmonic antennas, performed on a single photonic chip. This provides an in-depth analysis of the enhancement and holds promise to wavelength-multiplex multiple single-photon emitters on the same chip.

The photoluminescence (PL) lifetime $\gamma$ of an emitter in the gap of an antenna has a nonradiative and a radiative part

$$
\gamma=\gamma_{0, n r}+\gamma_{0, r}(\text { LDOS }- \text { LRDOS })+\gamma_{0, r} \text { LRDOS, }
$$

which in turn depend on the radiative part of the local density of states (LRDOS) and the initial decay rate $\gamma_{0}=\gamma_{0, r}+\gamma_{0, n r}$ of the emitter in absence of the antenna. In this work we used plasmonic bowtie antennas placed on silicon nitride waveguides to provide Purcell enhancement for IR emitting colloidal PbS/CdS QDs.

Further author information: (Send correspondence to L.E. or D.V.T)

L.E.: E-mail: lukas.elsinger@ugent.be

D.V.T.: E-mail: dries.vanthourhout@ugent.be

Photonic and Phononic Properties of Engineered Nanostructures X, edited by Ali Adibi, Shawn-Yu Lin, Axel Scherer, Proc. of SPIE Vol. 11289, 1128914 - @ 2020 SPIE · CCC code: $0277-786$ X/20/\$21' doi: 10.1117/12.2547265 


\section{DESIGN}

The false-color SEM micrograph in Figure 1a shows a bowtie antenna with colloidal PbS/CdS QD emitters placed the antenna gap, also used in our previous work. ${ }^{2}$ The emitters are excited from the top with a pulsed laser $(\lambda=700 \mathrm{~nm})$ and the spectrally broad QD emission (centered around $\lambda=1150 \mathrm{~nm}$ ) is collected and guided by the silicon nitride waveguide. Finite difference time domain (FDTD) simulation results of the $\operatorname{LRDOS}(\lambda)$ in the center of the gap of a plasmonic bowtie antenna (30 nm evaporated gold and a gap width of $25 \mathrm{~nm}$ ) can be found in Figure 5a. Figure 1b shows a false-color SEM top-view of a waveguide-coupled superconducting nanowire single-photon detector (SNSPD), that we use to efficiently detect incoming photons with low timing jitter. Simulations show that for a length $25 \mu \mathrm{m}$, the NbTiN nanowires absorb $98 \%$ of the radiation in the TE waveguide mode and $82 \%$ of the TM mode. We further estimated the internal detection efficiency of the SNSPD to be between $70 \%$ and $99 \%$ for typical devices and measured a timing jitter of $43 \pm 3$ ps and a recovery time of $9 \mathrm{~ns}$.

a

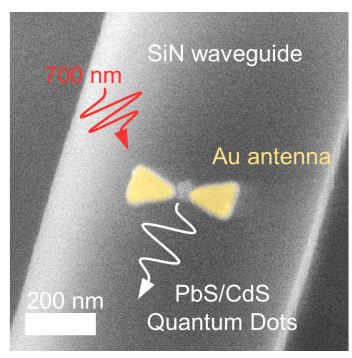

b

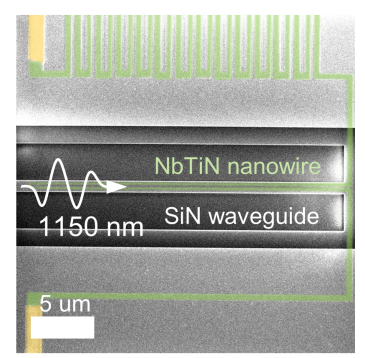

Figure 1. False-color SEM images of the fabricated structures. (a) Plasmonic bowtie antenna on top of a silicon nitride waveguide with collidal $\mathrm{PbS} / \mathrm{CdS}$ QDs placed in the center of the antenna gap. The emitters are pumped from the top with a pulsed laser $(\lambda=700 \mathrm{~nm})$, the emission (centered around $\lambda=1150 \mathrm{~nm}$ ) is guided on the chip through the silicon nitride waveguide. (b) Waveguide-coupled superconducting nanowire single-photon detectors (SNSPDs) enable the efficient detection of incoming photons with low timing jitter. The figure was adapted from our previous work. ${ }^{2}$

Next to integrated emitters and detectors, our silicon nitride photonic chips contain high-performance passive components as sketched in Figure 2. The use of low-fluorescence PECVD silicon nitride enables a waveguide loss below $1 \mathrm{~dB} / \mathrm{cm}$ and corrugated sidwall grating filters with a $-40 \mathrm{~dB}$ extinction were implemented, to remove the light of the pump laser from the waveguide. We further designed a 4-channel planar concave grating (PCG) spectrometer, matching the QD emission spectrum. The use of distributed Bragg reflectors (DBRs) as mirror facets allows for a high reflectivity despite the relatively low index contrast between silicon nitride and the cladding material. Narrow taper tips help to reduce reflections at the transition from the waveguide mode to the slab mode region of the spectrometer. Employing these combined measures enabled an insertion loss as low as $1.5 \mathrm{~dB}$ (see Figure 5d) for the spectrometer. In addition, we implemented absorbing metal lines next to the silicon nitride waveguides, in order to suppress stray light coupling, which can be a significant source of error in quantum photonic applications.

\section{FABRICATION}

Fabrication started from a blank silicon wafer, which was thermally oxidized to form the bottom cladding for the waveguides. Magnetron co-sputtering was used to deposit a $9.5 \mathrm{~nm} \mathrm{NbTiN}$ layer, before creating $\mathrm{Cr} / \mathrm{Au}$ contacts using electron-beam lithography and a lift-off process (see Figure 3). The U-shaped nanowires were patterned in a second electron-beam lithography step, using HSQ resist and dry etching in $\mathrm{SF}_{6}$ and $\mathrm{O}_{2}$ chemistry. The $70 \mathrm{~nm}$ wide nanowires were separated by a $200 \mathrm{~nm}$ gap and to avoid latching of the detector due to the small kinetic inductance, we included a $2.5 \mathrm{~mm}$ long and $400 \mathrm{~nm}$ wide section serving as a series inductor. By fabricating 

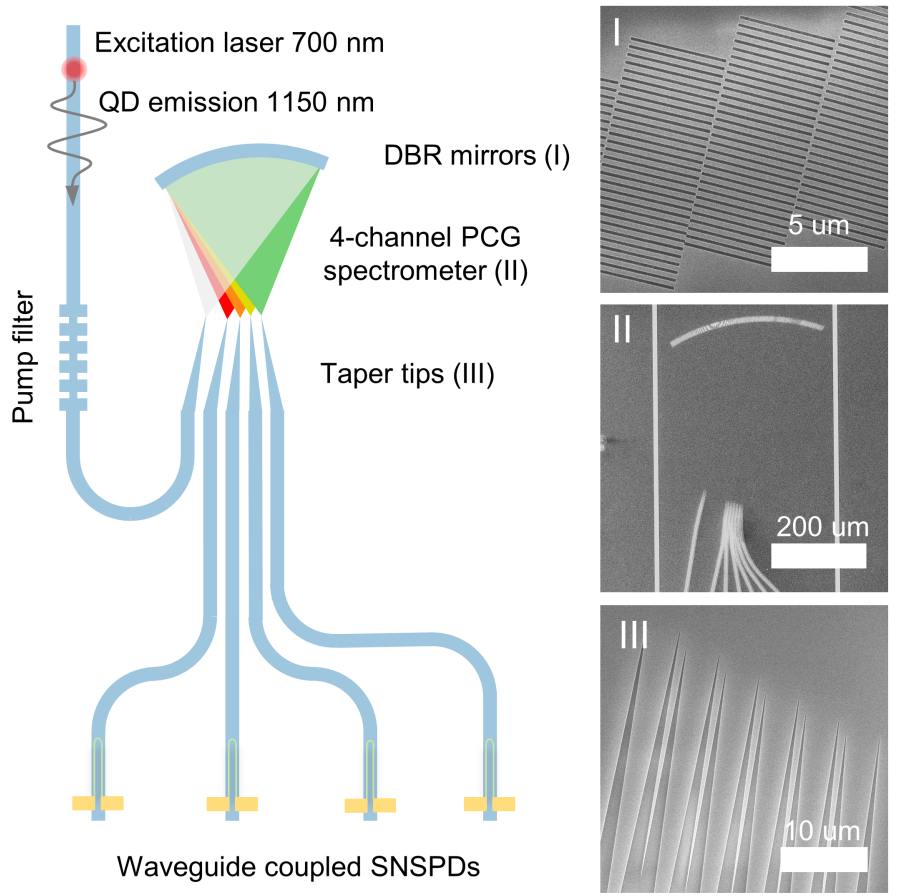

Figure 2. Sketch of the on-chip planar concave grating (PCG) spectrometer and insets showing SEM details of a fabricated device. (I) Distributed Bragg Reflector (DBR) elements of the grating mirror, providing a high reflectivity in the silicon nitride platform. (II) Top view of the PCG with metal lines placed next to it, intended to suppress stray light coupling. (III) Narrow taper tips reduce reflections at the transition from the waveguide mode to the slab mode, allowing for a low insertion loss of the spectrometer.

the SNSPDs first, it was possible to test and pre-select the devices, ${ }^{3}$ before the deposition of the $300 \mathrm{~nm}$ thick waveguide layer. Low-frequency plasma enhanced chemical vapor deposition (PECVD) was used for the silicon nitride layer, to minimize the fluorescence background at cryogenic temperatures, as well as the waveguide loss. Next, plasmonic bowtie antennas, additional alignment markers and the absorbing metal lines were fabricated using a third electron-beam lithography step and a lift-off process. A thin layer of $2 \mathrm{~nm}$ sputtered titanium was used to improve the adhesion of the $30 \mathrm{~nm}$ thick evaporated gold. In a fourth electron-beam lithography step the silicon nitride photonic components were defined using a positive resist (AR-P 6200 from Allresist) and reactive ion etching (RIE) in $\mathrm{CF}_{4}$ and $\mathrm{H}_{2}$ chemistry. To achieve a high overlay accuracy, we used an automatic marker recognition provided with the electron-beam lithography system (Raith Voyager). A final electron-beam lithography step and a lift-off process were used to selectively place the spin-coated PbS/CdS colloidal QDs in the $25 \mathrm{~nm}$ wide antenna gaps. The chips were subsequently encapsulated with a $1 \mu \mathrm{m}$ thick $\mathrm{SiO}_{2}$ top cladding, deposited by high-frequency PECVD at room temperature. Contact lithography and RIE in $\mathrm{CF}_{4}, \mathrm{SF}_{6}$ and $\mathrm{O}_{2}$ chemistry was used to open up the contact pads. The samples were then glued to a printed circuit board with silver paste and wire bonded to allow electrical biasing and readout of the detectors via RF coaxial cables suited for cryogenic environments. 


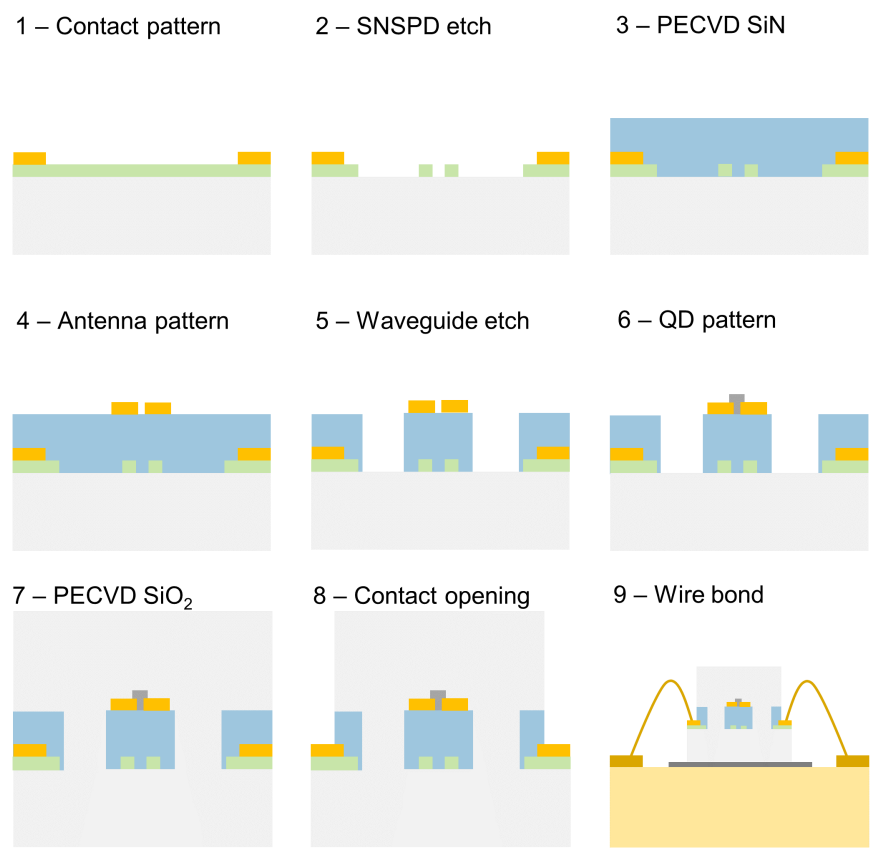

Figure 3. Overview of the main device fabrication steps. (1) Contacts are patterned on a chip coated with superconducting NbTiN. (2) Electron-beam lithography is used to pattern the U-shaped superconducting nanowire single-photon detectors (SNSPDs). (3) Low-fluorescence PECVD silicon nitride is deposited on the chip. (4) Plasmonic antennas and alignment markers are patterned using electron-beam lithography. (5) Waveguides are patterned with electron-beam lithography. (6) Colloidal PbS/CdS QDs are deposited in the gap of the plasmonic antennas using a lift-off process. (7) The whole chip is coated with PECVD silicon oxide to protect the waveguides outside of a cleanroom environment. (8) Contacts are opened using optical contact lithography. (9) The chip is bonded on a printed circuit board (PCB) using silver paste and electrical contacts are formed using wire bonds.

\section{MEASUREMENT}

All measurements were performed in a liquid helium bath cryostat (4.2 K base temperature) and PL decay traces were obtained from time correlated single photon counting (TCSPC), with the "start" event from the excitation laser and the "stop" event from the SNSPDs driver. We then fitted a stretched exponential

$$
c(t)=a e^{-\left(\frac{t}{\tau_{K}}\right)^{\varsigma}}
$$

to the normalized data, after subtraction of the background. This accounts for a distribution of decay times for $\zeta<1$ and turns into an exponential decay expected for a single-exciton emission at $\zeta=1$. To judge the effect of the plasmonic antennas on the QD PL lifetime, we then extracted the mean lifetime $\bar{\tau}$ from the fitted parameters according to

$$
\bar{\tau}=\frac{\tau_{K}}{\zeta} \Gamma\left(\frac{1}{\zeta}\right)
$$

using the gamma function $\Gamma$. 

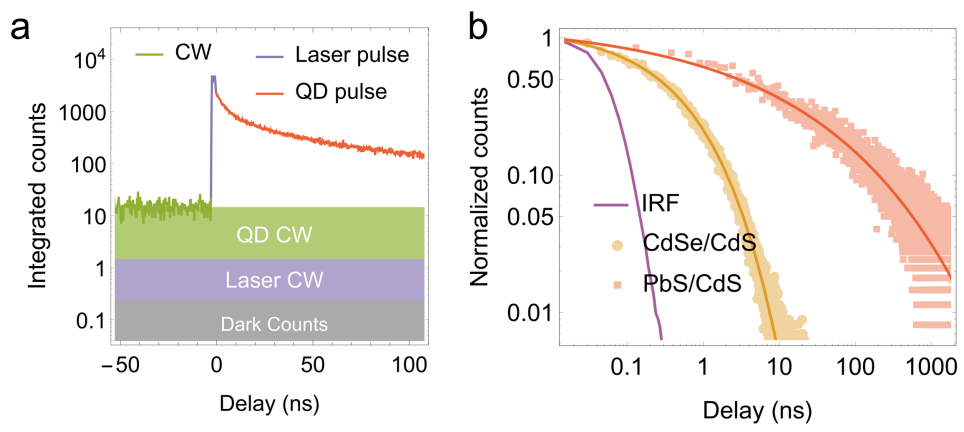

Figure 4. (a) Typical integrated counts obtained from time correlating detection events and excitation pulses. We can identify the excitation laser pulse as well as the QD PL decay in the trace. (b) Typical normalized traces after background subtraction measured for CdSe/CdS and PbS/CdS colloidal QDs fitted with a stretched exponential and the instrument response function (IRF) of the chip-based system.

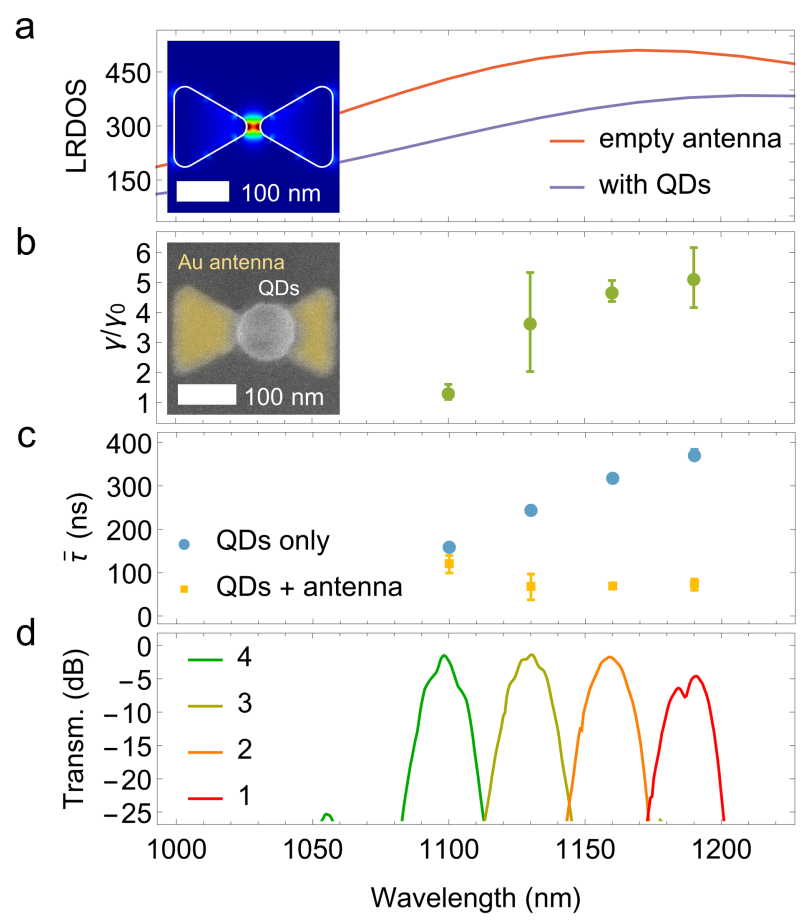

Figure 5. Simulated radiative local density of states (LRDOS) in the gap of a plasmonic antenna (a) with the inset showing a corresponding 2D map for a wavelength of $\lambda=1150 \mathrm{~nm}$. Rate enhancement measured using the four channels of an onchip spectrometer (b) and an inset showing a false-color SEM image of a fabricated structure. Average lifetimes extracted from a stretched exponential fit of the PL decay (c) and the transmission measured through the four spectrometer channels (d). Error bars represent 1- $\sigma$ confidence intervals.

Figure 4a shows a typical integrated trace, where we can identify the pulsed contribution from the excitation laser and the QD PL decay. The background consists of the detector dark counts, as well as continuous wave $(\mathrm{CW})$ contributions from the excitation laser and the QDs. Figure 4b shows the data after subtraction of the 
background and normalization on a double-logarithmic scale. The instrument response function (IRF) of our chip-based system is compared to the PL decay of CdSe/CdS QDs emitting in the visible (excitation with $\lambda=$ $400 \mathrm{~nm}$ ) and the IR-emitting PbS/CdS QDs used in this study. The traces in Figure 4 were obtained without the use of the on-chip spectrometer and represent an average over the whole QD emission spectrum.

Using the on-chip spectrometer we extracted the average lifetimes $\bar{\tau}$ displayed in Figure $5 \mathrm{c}$ for the different channels. For reference measurements in absence of the plasmonic antennas, we used larger patches of QDs placed on a different waveguide, to obtain enough signal at the detectors. Therefore, it was not possible to quantify the enhancement of the emitter quantum yield $\phi / \phi_{0}$ in this experiment. Using the antennas, the spectral dependence of the average lifetimes $\bar{\tau}$ changes compared to the isolated QDs on a waveguide, already reported in our previous work. ${ }^{2}$ The extracted rate enhancement $\gamma / \gamma_{0}$ in Figure $5 \mathrm{~b}$ shows a clear wavelength dependence, with the largest value recorded for the channel centered at $1190 \mathrm{~nm}$. This is in agreement with FDTD simulations of the $\operatorname{LRDOS}(\lambda) \propto \gamma / \gamma_{0}$, which show a peak at $1150 \mathrm{~nm}$ for an empty antenna (Figure 5a). However, there is a red-shift of the resonance when the QD pillar is included in the simulations, using refractive index values from ellipsometry measurements. Also, the wavelength-dependent simulation in Figure 5a were only performed for the center of the antenna gap. To match the conditions of the experiment, $\operatorname{LRDOS}(\lambda)$ has to be averaged over the whole QD pillar. While for an ideal emitter

$$
\operatorname{LRDOS}=\frac{\phi}{\phi_{0}} \frac{\gamma}{\gamma_{0}}
$$

can be approximated by the rate enhancement, the PbS/CdS QDs used in this experiment had PL quantum yield below $1 \%$ after the extensive processing used to place them on the photonic chip, ${ }^{2}$ which explains a further reduction compared to the simulations.

\section{CONCLUSION AND OUTLOOK}

In summary, we showed first wavelength-resolved measurements of the Purcell enhancement of QDs deterministically positioned in the gap of plasmonic antennas, performed on a single photonic chip. Through a careful analysis of the different contributions to the count rate at the SNSPDs we predict our method to scale down to single QDs. ${ }^{2}$ In addition, newly developed emitters which meet quantum optics requirements can be readily integrated on the chip-based platform. Next to potential applications in integrated quantum optics, this enables lifetime-spectroscopy of solution-processed nano-materials at cryogenic temperatures, using a single photonic chip.

\section{ACKNOWLEDGMENTS}

This work was supported by the European Commission via the Marie-Sklodowska Curie action Phonsi (H2020MSCA-ITN-642656). D.V.T. and Z.H. acknowledge the FWO-Vlaanderen for supporting this research (project nr. G087317N). R.G. thanks René Van Der Molen, Andréas Fognini and Johannes Los for technical support and beneficial discussions. I.E.Z. acknowledges the support of NWO LIFT-HTSM for Physics 2016-2017, project nr. 680-91-202 and support from Single Quantum B.V. (SQ).

\section{REFERENCES}

[1] Koenderink, A. F., "Single-Photon Nanoantennas," ACS Photonics 4(4), 710-722 (2017).

[2] Elsinger, L., Gourgues, R., Zadeh, I. E., Maes, J., Guardiani, A., Bulgarini, G., Pereira, S. F., Dorenbos, S. N., Zwiller, V., Hens, Z., and Van Thourhout, D., "Integration of Colloidal PbS/CdS Quantum Dots with Plasmonic Antennas and Superconducting Detectors on a Silicon Nitride Photonic Platform," Nano Lett. , acs.nanolett.9b01948 (jul 2019).

[3] Gourgues, R., Zadeh, I. E., Elshaari, A. W., Bulgarini, G., Los, J. W. N., Zichi, J., Dalacu, D., Poole, P. J., Dorenbos, S. N., and Zwiller, V., "Controlled integration of selected detectors and emitters in photonic integrated circuits," Opt. Express 27, 3710 (feb 2019). 